\title{
Decreasing False-Positive RFID Tag Reads by Improved Portal Antenna Setups
}

\author{
Thorben Keller*, Frédéric Thiesse ${ }^{\dagger}$, Alexander Ilic ${ }^{\ddagger}$ and Elgar Fleisch ${ }^{\S}$ \\ *University of St. Gallen \\ St. Gallen, Switzerland \\ Email: thorben.keller@unisg.ch \\ tUniversity of Wuerzburg \\ Würzburg, Germany \\ Email: frederic.thiesse@uni-wuerzburg.de \\ $\ddagger$ University of St. Gallen \\ St. Gallen, Switzerland \\ Email: alexander.ilic@unisg.ch \\ $\S$ University of St. Gallen \& ETH Zurich \\ St. Gallen \& Zurich, Switzerland \\ Email: elgar.fleisch@unisg.ch
}

\begin{abstract}
The problem of false-positive RFID tag reads i.e., tags that have been read unintentionally by a reader, is crucial for the actual implementation of RFID solutions in the real world. Prior research has shown that the use of the low-level reader data is suitable to approach this problem and it is the scope of this paper to evaluate the use of alternative RFID reader antenna setups to support this idea. We collected a large number of data in a productive RFID enabled distribution center, derived additional knowledge from our findings and used it to train a machine learning algorithm for the detection of such false-positive reads. It will be shown that the proposed solutions significantly improve the previously in the literature presented classification algorithms.
\end{abstract}

\section{INTRODUCTION}

In recent years, the application of Radio Frequency Identification (RFID) technology in supply chain management has attracted the interest of several industries worldwide [1]. This development has been strongly driven by standardization activities, cost erosion, and the miniaturization of microelectronic components [2], [3].

The long-term objective of using RFID in the supply chain is the automatic identification, tracing, and verification of goods along their way from the supplier to the customer. The prerequisite for such an all-embracing process is that the supplier or the producer at the starting point of the supply chain attaches RFID tags to all of their products. This would allow the unique identification of every single product from production to consumption, thus making it much easier, for example, to trace the origin of contaminated food lots or to record the history of a pharmaceutical's buyers and sellers throughout the supply chain [4].

One of the key processes in many supply chains is the shipping from distribution centers where a highly efficient warehouse management is essential to ensure a smooth flow of goods to the stores. The goods, already tagged with RFID transponders at some preceding step in the supply chain, are

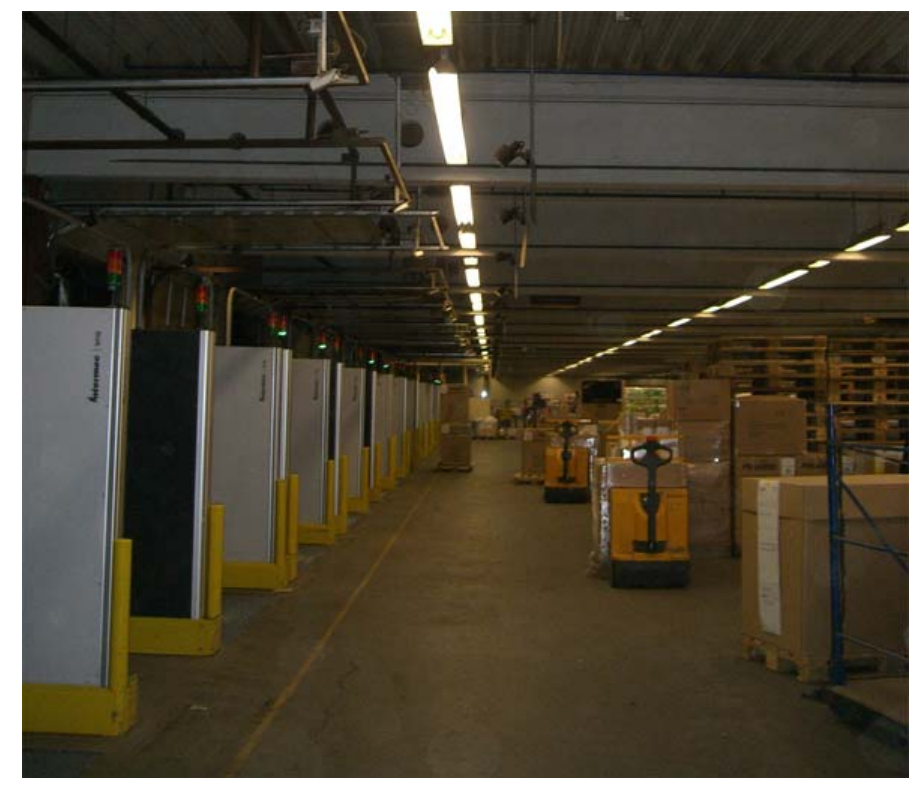

Fig. 1. RFID portals at shipment dock doors in a distribution center.

automatically detected when loaded into a truck. As soon as the warehouseman passes through the portal with a pallet on his forklift, the tag together with the corresponding EPC is automatically scanned and the information about the loading of the pallet is forwarded to the warehouse management system. This contrasts with a bar code based system where each pallet has to be scanned manually by the warehouseman. At this point, any discrepancies between the expected and the actually sent goods are automatically detected.

\section{A. Statement of the Problem}

However, in [5] it was shown that the problem of falsepositive RFID tag reads hampers a productive roll-out of 
such a solution in a logistic environment. Because the portal antennas are not directional and have a read range of several meters, not only is the pallet moving through the portal detected, but any other in range as well. The problem is that readers cannot distinguish between pallets that are loaded into trucks and those that appear in the RF field by accident. If all pallets were reported to the warehouse management system as being shipped, the resulting incorrect invoices would mean stores have to pay for goods that they neither ordered nor received. Furthermore, returning wrongly shipped pallets back to the the sender incurs very high costs that can often exceed the actual value of the goods. It is evident therefore that as long as this problem remains unsolved, a reliable and productive use of RFID technology in distribution center processes is not feasible. This description of an RFID-enabled outgoing goods scenario leads to the understanding that there is a fundamental difference between the tag of interest and the false-positives: the loaded pallet hast obviously been moved through the portal while any false-positive pallet has not moved and is still somewhere in the read range of the RFID antennas. Therefore, for the sake of simplicity, throughout the rest of this paper the pallet that was loaded into a container is denoted a moved pallet and a false-positive read is denoted a static pallet.

\section{B. Related Work}

The detection of false positive RFID tag reads is a complex challenge to the technology in many applications as stated for example in [6] and [7]. As of today, only few approaches were presented in the literature to deal with this problem but they often consider it together with the occurrence of falsenegative tag reads, for example in [8]. In [9] and [10], a sliding window approach is used to filter the RFID data stream. If a tag is read less often than a given threshold during the sliding time window it is considered to be a false-positive. Because individual tags are filtered out on the low-level reader level this might lead to an increasing number of false-negatives. Thus, a balance has to be found between the readability of tags and the detection of false positives as studied in [11].

The use of additional hardware is proposed in [12] and [13]. The authors propose to determine the presence or absence of tagged objects at arbitrary locations in the supply chain. For this purpose 2 readers (i.e., one additional) are installed at each read point. If a tag is read at both readers then it is classified as present and absent if none of the readers recognized it. If only one of the readers reads the tag, a sliding window approach is used to determine the presence or absence of the tag. An advanced approach makes use of an additional tag attached to the object that has to be read by the readers as well.

The most advanced approach however was presented in [5] where it was shown that the low-level reader data is suitable to distinguish between moved and static pallets thus allowing to build a classification model to detect false-positive RFID tag reads. The classification algorithms described in this paper make use of the low-level reader data on the one hand and of a more advanced reader antenna setup on the other hand.

\section{PoRTAl Types}

The general recognition procedure of RFID tagged pallets is as follows. As soon as a warehouseman approaches the portal and walks into the truck this is recognized by a motion sensor and the reader antennas start scanning for pallets in range. Using the detection algorithms described below each pallet is being evaluated as either being moved or static. At some point the motion sensor recognizes that the warehouseman has left the truck and signals the reader to stop scanning. Three different portal antenna setups Standard Portals, Satellite Portals and Transition Portals will be described each of them with their own logic as to how moved and static pallets might be distinguished.

\section{A. Standard Portals}

The most commonly used type of RFID portal in the distribution center is the Standard Portal; its general antenna configuration is shown in Fig. 2a. A single reader is used with four different antennas (called Main Antennas) attached to it, two at each side of the portal, on top of each other and face to face with the other two. Antenna \#1 is located bottomleft, antenna \#2 bottom right, antennas \#3 and \#4 are located top left and right, respectively. As soon as the warehousman approaches the portal with a pallet all four antennas start scanning simultaneously until the stop trigger is activated.

\section{B. Satellite Portals}

Satellite Portals are an advanced version of the Standard Portals which use an additional RFID reader with 4 more antennas. Two of these antennas are directed toward the distribution center (antennas \#7 and \#8) and the other two (antennas \#5 and \#6) are directed toward the truck - as can be seen in Fig. 2b. The four remaining correspond to the antennas also used in the Standard Portals and are thus still referred to as Main Antennas. The additional antennas are denoted $D C$ Antennas and the other two are denoted Truck Antennas.

As soon as the warehouseman approaches a Satellite Portal the four Main Antennas and the two Truck Antennas start scanning simultaneously and every single tag detection is recorded until the stop trigger is activated. At that moment the DC Antennas start scanning for transponders still present in the distribution center. The idea behind this is that a tag that moved through the portal is expected to be inside the container rather than inside the distribution center. Consequently, all reads of tags that can be seen in the distribution center after the end of the actual detection period should be considered falsepositives. The logic behind this antenna configuration leads to 7 disjunct cases that can apply to a transponder detected during a specific loading.

Case 1The tag has been read by Main-, Truck- and DC Antennas. This first case is interesting: this is a situation where after the loading of a pallet has finished reflections can cause an effect where a tag can still be seen inside the distribution center even although it had previously moved through the portal 


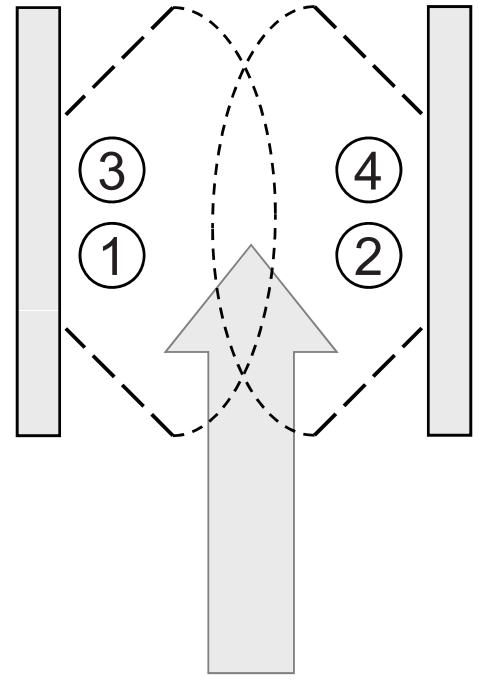

(a)

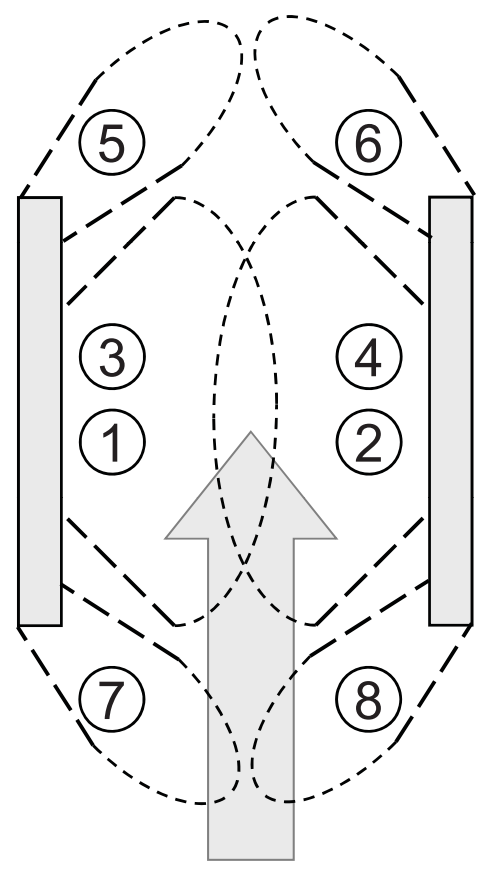

(b)

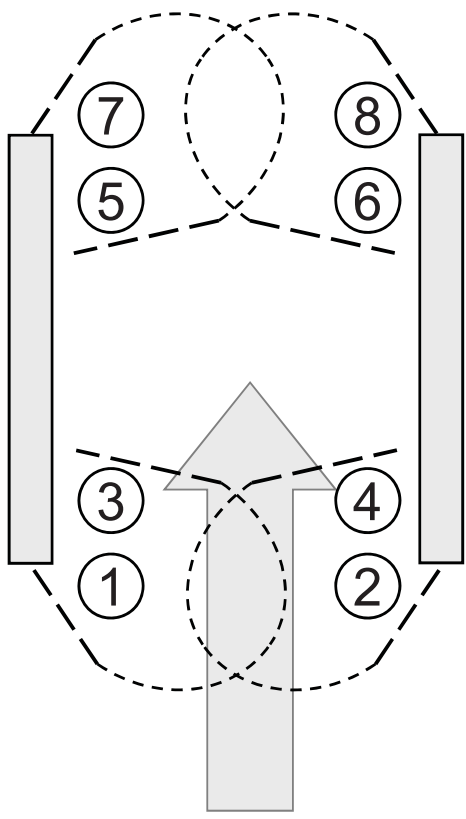

(c)

Fig. 2. Antenna orientation of Standard-, Satellite- and Transition Portals. The movement of a pallet is indicated by the gray arrow directing from the distribution center to the truck.

and into the truck. Tags that this case applies to are most likely false-positives.

Case 2The tag has been read by DC- and Main Antennas only. This means that the tag has been seen during the loading but afterwards appears to be still in the distribution center. Tags in this case are expected to be static, i.e., false-positives.

Case 3The tag has been read by DC- and Truck Antennas but not by the Main Antennas. This case is similar to Case 1 since reflections cause the effect that the tag appears to be in two different locations. These tags are expected to be false-positives.

Case 4The tag has been read by the DC Antennas only. Since the tag has neither been read by the Main- nor by the Truck Antennas, it has likely not been moved through the portal and thus is a false-positive.

Case 5The tag has been read by Main- and Truck Antennas. This is like the first case, where a tag is expected to have been loaded into the container because it was seen during the loading by Main- and Truck Antennas but afterwards the DC Antennas signal that it is not in the distribution center anymore.

Case 6The tag has been read by the Main Antennas only. This means that a tag has been read during the loading but then suddenly vanishes. Nevertheless the tag has apparently passed through the portal and is thus expected to be in the container.

Case 7The tag has been read by the Truck Antennas only. These tags have not been read during the loading and consequently it is most likely that they have been loaded at a previous point in time and thus are falsepositives with regard to moving.

\section{Transition Portals}

Like the Standard Portals the Transition Portals make use of two different readers, but they do not have any Main Antennas (see Figure 2c). The general idea is that the 4 antennas of the first reader (antennas \#1-\#4) are directed toward the distribution center and the 4 antennas of the second reader (antennas \#5-\#8) are directed towards the truck. Note that similar to the Main Antennas antennas \#1, \#2, \#5 and \#6 are installed on top of antennas \#3, \#4, \#7 and \#8, respectively. As soon as a warehouseman approaches the portal the DC- and Truck Antennas begin scanning simultaneously and every single tag detection is recorded until the stop trigger is activated and the scan is terminated. The idea behind this is that a tag that has been moved though the portal is expected to be seen first by the DC antennas and afterwards by the Truck Antennas. Accordingly, all tags seen only by the DC- or only by the Truck Antennas should be considered false-positive reads. The 
logic behind this antenna configuration leads to the following 3 disjunctive cases.

Case 1The tag has been read by the DC Antennas only. Since it has not been read by the Truck Antennas it is likely to be a false-positive tag.

Case 2The tag has been read by the Truck Antennas only. Similar to Case 1 it is likely to be a false-positive.

Case 3The tag has been read by the DC- Antennas as well as by the Truck Antennas. It is most likely that this tag has been moved through the portal and thus has been loaded into the truck.

\section{DATA COLlection}

The data set used in this paper to evaluate the different approaches was collected in a productive environment and under real world conditions at the METRO Group central distribution center in Unna, Germany. This center sees between 3,500 and 8,000 pallet movements a day and all of the shipment dock doors have been equipped with RFID portals (79 Standard-, 4 Satellite- and 8 Transition Portals) to automatically register any outgoing pallets. In order to obtain the required sample data set we accompanied warehousman and monitored the loading of pallets from the distribution center into containers. The task was to keep track of which pallets that were recognized by the RFID reader during the loading process had actually been moved through the outgoing goods portal and also those which were present in the reading field of the portal antennas only by accident.

On the basis of the three different portal types described above three major data sets are compiled. In principle, each portal type is represented by its own data set. However, Satellite- and Transition Portals lead to different cases that transponders can apply to, so these in turn are interpreted as distinct sub data sets. Table I shows the portal types including possible cases, the particular antennas that read the tag and the denomination of the respective data sets.

TABLE I

Data Set DenOmination

\begin{tabular}{lll}
\hline Portal Type & Involved Antennas & Data Set \\
\hline Standard Portals & Main & STD_COMPLETE \\
\hline Satellite Portals & Union of cases 1-7 & SAT_COMPLETE \\
Case 1 & Main-, Truck- and DC & SAT_ALL \\
Case 2 & DC- and Main & SAT_DC_MAIN \\
Case 3 & DC- and Truck & SAT_DC_TRUCK \\
Case 4 & DC Antennas only & SAT_DC_ONLY \\
Case 5 & Main- and Truck & SAT_MAIN_TRUCK \\
Case 6 & Main Antennas only & SAT_MAIN_ONLY \\
Case 7 & Truck Antennas only & SAT_TRUCK_ONLY \\
\hline Transition Portals & Union of cases 1-3 & TRA_COMPLETE \\
Case 1 & DC Antennas only & TRA_DC_ONLY \\
Case 2 & Truck Antennas only & TRA_TRUCK_ONLY \\
Case 2 & DC- and Truck & TRA_BOTH \\
\hline
\end{tabular}

\section{A. Standard Portal data description}

The number of moved and static tags monitored at the Standard Portals including the resulting false-positive rate is shown in Table II.
TABLE II

Monitored Pallets at the Standard Portals

\begin{tabular}{lrrrr}
\hline Data Set & Moved & Static & Total & FP-Rate \\
\hline STD_COMP LETE & 13,245 & 40,743 & 53,988 & $75.47 \%$ \\
\hline
\end{tabular}

A total of 53,988 pallets were observed, of which 40,743 were false-positives. This meant that on average there are slightly more than 3 static tags per moved tag read during a loading which corresponds to a false-positive rate of $75.47 \%$. It is obvious then, that in order to attain a reliable and fully functional RFID enabled outgoing goods process, these falsepositives need to be filtered out.

\section{B. Satellite Portal data description}

The number of moved and static tags monitored at the Satellite Portals, including the false-positive rate, is shown in Table III. Based upon the possible cases defined above the respective data is shown for each individual sub data set.

A total of 14,777 pallets were monitored, of which 12,806 were false-positives. This means that on average there are almost 6.5 static tags per moved tag read during a loading which corresponds to a false-positive rate of $86.66 \%$. The general idea of the Satellite Portals was that tags that were read by the DC Antennas are expected to be static. These tags can be found in the following data sets:

- SAT_ALL,

- SAT_DC_MAIN,

- SAT_DC_TRUCK and

- SAT_DC_ONLY.

In sum there are 3,247 tags in these data sets of which 3,225 were static. This corresponds to a false-positive rate of $99.32 \%$. Of the 5,122 tags that were read only by the Truck Antennas $99.79 \%$ were false-positives. Because of the very high false-positive rate, each tag that applies to one of the above cases is automatically considered to be a false-positive. The remaining data sets of interest are

- SAT_MAIN_TRUCK and

- SAT_MAIN_ONLY.

For these tags only, a classification model needs to be constructed.

TABLE III

Monitored Pallets at the Satellite Portals

\begin{tabular}{lrrrr}
\hline Data Set & Moved & Static & Total & FP-Rate \\
\hline SAT_ALL & 10 & 279 & 289 & $96.54 \%$ \\
SAT_DC_MAIN & 12 & 784 & 796 & $98.49 \%$ \\
SAT_DC_TRUCK & 0 & 40 & 40 & $100.00 \%$ \\
SAT_DC_ONLY & 0 & 2,122 & 2,122 & $100.00 \%$ \\
SAT_MAIN_TRUCK & 1,282 & 1,899 & 3,181 & $59.70 \%$ \\
SAT_MAIN_ONLY & 656 & 2,571 & 3,227 & $79.67 \%$ \\
SAT_TRUCK_ONLY & 11 & 5,111 & 5,122 & $99.79 \%$ \\
\hline SAT_COMPLETE & 1,971 & 12,806 & 14,777 & $86.66 \%$ \\
\hline
\end{tabular}


TABLE IV

Monitored Pallets at the Transition Portals

\begin{tabular}{lrrrr}
\hline Data Set & Moved & Static & Total & FP-Rate \\
\hline TRA_DC_ONLY & 28 & 5,779 & 5,807 & $99.52 \%$ \\
TRA_TRUCK_ONLY & 31 & 4,316 & 4,347 & $99.29 \%$ \\
TRA_BOTH & 1,299 & 2,392 & 3,691 & $64.81 \%$ \\
\hline TRA_COMPLETE & 1,358 & 12,487 & 13,845 & $90.19 \%$ \\
\hline
\end{tabular}

\section{Transition Portal data description}

The number of moved and static tags monitored at the Transition Portals, including the false-positive rate, is shown in Table IV. Based upon the possible cases the respective data is shown for each individual sub data set.

A total of 13,845 pallets were observed, of which 12,487 were false-positives. This means that on average there are around 9.2 static tags per moved tag read during a loading which corresponds to a false-positive rate of $90.19 \%$. The general idea of the Transition Portals is that transponders that were seen either by only the DC- or only by the Truck Antennas are expected to be static. These tags can be found in the

- TRA_DC_ONLY and

- TRA_TRUCK_ONLY

data sets. In sum there are 10,154 reads in these data sets of which 10,095 were static. This corresponds to a false-positive rate of $99.42 \%$. Because of the very high false-positive rate, each tag that applies to one of the above cases is automatically considered to be a false-positive.

The remaining data set of interest is

- TRA_BOTH.

For these tags only, a classification model needs to be constructed.

\section{The Final Data Sets}

The separation of the monitored data into distinct subsets revealed a number of cases where the application of a classification model is unnecessary because of the very high falsepositive rate. For example, every single one of the 2,122 tags that were detected only by the DC Antennas of the Satellite Portals were false-positives, thus tags corresponding to this case can be classified as "static" by definition. The relevant data sets for which a classification model needs to be generated are shown in Table V.

TABLE V

Sample Data in the Relevant Data SeTs

\begin{tabular}{lrrrr}
\hline Data Set & Moved & Static & Total & FP-Rate \\
\hline STD_COMPLETE & 13,245 & 40,743 & 53,988 & $75.47 \%$ \\
SAT_MAIN_TRUCK & 1,282 & 1,899 & 3,181 & $59.70 \%$ \\
SAT_MAIN_ONLY & 656 & 2,571 & 3,227 & $79.67 \%$ \\
TRA_BOTH & 1,299 & 2,392 & 3,691 & $64.81 \%$ \\
\hline
\end{tabular}

\section{Classification Model Building}

As stated above we want to train a classification model that enables us to distinguish between moved and static pallets. We used standard data mining software called RapidMiner [14] to build a decision tree based on the attributes presented in [5] to predict the pallet type. Based on the antenna setup logic of the Satellite- and Transition Portal setup specific attributes are derived for these and added to the decision tree learner to improve the classification algorithms. We furthermore used boosting and various feature generation approaches to obtain an even larger number of attributes.

\section{A. General Attributes}

As has been shown in [5] the examination of certain characteristics of the low-level reader data can be helpful for the detection of false-positive RFID tag reads. Those attributes can be separated into 3 different subsets depending on the type of low-level data being used.

First of all there are the attributes derived from the Received Signal Strength Indication (RSSI) which is a measurement of the power of the received signal strength the tag emits. Higher RSSI values indicate a close distance between sender and receiver, lower RSSI values indicate a farther distance. Meaningful attributes to describe a pallet include the minimum, maximum, mean and standard deviation of the received signal strength.

The second group of attributes was called SinceStart Attributes and they correspond to the time that has passed between the start of the scanning procedure and the actual detection of the tag. Meaningful attributes include the minimum and maximum time that has passed as well as the difference between these timestamps i.e., the period of time over which the tag has been read.

The last group of attributes took the knowledge about which of the involved antennas actually read the tag and how often that happened.

As has been proven the evaluation of those attributes already leads to a powerful tool for the detection of false-positive tag reads. However, using the alternative antenna design described above we are able to create a number of additional attributes specific to the particular portal type. These attributes are described in the following subsections.

\section{B. Satellite Portal specific Attributes}

Satellite Portals consist of Main- (MA) as well as Truck(TA) and DC (DC) Antennas as described before. Because moved tags pass the portal it is expected that they are read first by the Main- and subsequently by the Truck Antennas (remember that if they are read by the DC Antennas after the loading then they are classified as static by definition). Accordingly, a number of additional characteristics are introduced to map the order at which it was read against meaningful attributes. From all possible cases, only the tags read by both Main- and Truck Antennas are of interest at this point because for tags read only by the Main Antennas no order can be defined and all other cases are automatically classified as static by definition. 
TABLE VI

SATELLITE PORTAL SPECIFIC ATTRIBUTES

\begin{tabular}{|c|c|c|}
\hline Name & Val. & Condition \\
\hline \multirow{2}{*}{ Seen $_{\text {First }}$} & M & First detection of tag at MA \\
\hline & $\mathrm{T}$ & First detection of tag occurred at TA \\
\hline \multirow{2}{*}{ Seen $_{\text {Last }}$} & $\mathrm{M}$ & Last detection of tag at MA \\
\hline & $\mathrm{T}$ & Last detection of tag occurred at TA \\
\hline \multirow{2}{*}{ Seen $_{\text {Longer }}$} & M & MA read over longer period of time \\
\hline & $\mathrm{T}$ & TA read over longer period of time \\
\hline \multirow{2}{*}{ First $_{M}$ Last $_{T}$} & 1 & First detection at MA, last detection at TA \\
\hline & 0 & Otherwise \\
\hline \multirow{2}{*}{ First $_{T}$ Last $_{M}$} & 1 & First detection at TA, last detection at MA \\
\hline & 0 & Otherwise \\
\hline \multirow{2}{*}{ First $_{M}$ Last $_{M}$} & 1 & First and last detection occurred at MA \\
\hline & 0 & Otherwise \\
\hline \multirow{2}{*}{ First $_{T}$ Last $_{T}$} & 1 & First and last detection occurred at TA \\
\hline & 0 & Otherwise \\
\hline \multirow{2}{*}{ Disjoint $_{M, T}$} & 1 & First det. at TA after last det. at MA \\
\hline & 0 & Otherwise \\
\hline \multirow{2}{*}{ Disjoint $_{T, M}$} & 1 & First det. at MA after last det. of TA \\
\hline & 0 & otherwise \\
\hline
\end{tabular}

The Satellite Portal specific attributes are shown in Table VI including the possible values and the respective conditions that have to be met.

\section{Transition Portal specific Attributes}

Transition Portals consist of DC- (DC) and Truck Antennas (TA). Because moved tags pass the portal it is expected that they are read first by the DC- and subsequently by the Truck Antennas (if they are read only by one of them they are classified as static by definition). Consequently, similar to the Satellite Portals a number of additional attributes are introduced to map the order at which a tag has been read. The Transition Portal specific attributes are shown in Table VII.

\section{RESULTS}

In order to estimate the relevance of the Satellite- and Transition Portal specific attributes, the value distribution is presented together with the precision and the recall values in Tables VIII and IX . Precision refers to the predictive power of a specific attribute value. For example, if a pallet has been detect by the Truck Antennas prior to the Main Antennas $\left(\right.$ Seen $_{\text {First }}=T$ in Table VIII)then we know with a probability of $93.6 \%$ that is a pallet that has not been loaded but is a falsepositive instead. Recall gives insight into which value is more typical for a specific pallet type (i.e., moved or static pallets). For example, we observe that $95.9 \%$ of the moved pallets were last detected by the Truck Antennas.

\section{A. Portal specific attribute Evaluation}

Remember that from all pallets monitored at the Satellite Portals only the pallets that have been detected either only by the Main Antennas (3,227 pallets) or by both Main- and Truck Antennas (3,181 pallets) require an algorithm to filter out falsepositive RFID tag reads and only in the latter case the portal
TABLE VII

TRANSITION PORTAL SPECIFIC ATTRIBUTES

\begin{tabular}{l|l|l}
\hline Name & Val. & Condition \\
\hline \multirow{2}{*}{ First } \\
& $\mathrm{D}$ & First detection of tag at DC \\
& $\mathrm{T}$ & First detection of tag at TA \\
\hline \multirow{2}{*}{ Last $_{\text {Seen }}$} & $\mathrm{D}$ & Last detection of tag at DC \\
& $\mathrm{T}$ & Last detection of tag at TA \\
\hline \multirow{2}{*}{ Seen $_{\text {Longer }}$} & $\mathrm{D}$ & DC read over a longer period of time \\
& $\mathrm{T}$ & TA read over a longer period of time \\
\hline \multirow{2}{*}{ First $_{D}$ Last $_{T}$} & 1 & First detection at DC, last detection at TA \\
& 0 & Otherwise \\
\hline \multirow{2}{*}{ First $_{T}$ Last $_{D}$} & 1 & First detection at TA, last detection at DC \\
& 0 & Otherwise \\
\hline \multirow{2}{*}{ First $_{D}$ Last $_{D}$} & 1 & First and last detection at DC \\
& 0 & Otherwise \\
\hline \multirow{2}{*}{ First $_{T}$ Last $_{T}$} & 1 & First and last detection at TA \\
& 0 & Otherwise \\
\hline Disjoint $_{D, T}$ & 1 & First det. at TA after last det. at DC \\
\hline Disjoint $_{T, D}$ & 0 & Otherwise \\
\hline
\end{tabular}

specific attributes apply. As can be seen in Table VIII some of the attributes (e.g., Seen First $_{\text {, First }}$ Last $_{T}$, First $_{T}$ Last $_{M}$, First $_{T}$ Last $_{T}$, Disjoint $(T, M)$ have a very high precision value and thus already allow to understand very well which attributes are useful to filter out false-positive tag reads.

Table IX shows the attribute value distribution of the Transition Portal specific attributes. Here as well some of the attributes show very well which attributes are probably most suitable for a false-positive detection algorithm. Remember that those attributes apply only to the pallets that have been detected by both DC- and Truck Antennas.

\section{B. Detection Algorithm Results}

We used the portal specific attributes together with the lowlevel reader data attributes (RSSI, SinceStart attributes) and a feature generation algorithm to train decision tree based classification algorithms. The results of the algorithms for the three portal types can be found in Table X. The quality of the approaches is measured using the classification accuracy, i.e., the fraction of tags correctly classified as moved or static. It can be observed that the use of the more advanced portal types brings a significant improvement over the use of the standard portals for both detection of moved and detection of static pallets. The error rate for detecting moved pallets at the Satellite Portals is only $2.59 \%$ compared to $5.34 \%$ at the Standard Portals which is an improvement of about $48 \%$. Static pallets can be detected best using the Transition Portals with an error rate of only $0.48 \%$ compared to the $1.49 \%$ error rate at the Standard Portals. The overall classification error is least for the Satellite Portals with only $0.96 \%$. This means less than 1 out of 100 detected pallets will be classified incorrectly using the Satellite Portals which is an improvement of more than 32\% compared to the Standard Portals. 
TABLE VIII

Satellite Portal Attribute Value Distribution

\begin{tabular}{|c|c|c|c|c|}
\hline Attribute & Value & Moved & Static & Precision \\
\hline \multirow{3}{*}{ Seen $_{\text {First }}$} & $\mathrm{M}$ & 1,195 & 629 & $65.5 \%$ \\
\hline & $\mathrm{T}$ & 87 & 1,270 & $93.6 \%$ \\
\hline & Recall & $93.2 \%$ & $66.9 \%$ & \\
\hline \multirow{3}{*}{ Seen $_{\text {Last }}$} & $\mathrm{M}$ & 53 & 310 & $85.4 \%$ \\
\hline & $\mathrm{T}$ & 1,229 & 1,589 & $56.4 \%$ \\
\hline & Recall & $95.9 \%$ & $83.7 \%$ & \\
\hline \multirow{3}{*}{ Seen $_{\text {Longer }}$} & $\mathrm{M}$ & 420 & 328 & $56.1 \%$ \\
\hline & $\mathrm{T}$ & 862 & 1,571 & $64.6 \%$ \\
\hline & Recall & $67.2 \%$ & $82.7 \%$ & \\
\hline \multirow{3}{*}{ First $_{M}$ Last $_{T}$} & 1 & 1,153 & 499 & $69.8 \%$ \\
\hline & 0 & 129 & 1,400 & $91.6 \%$ \\
\hline & Recall & $89.9 \%$ & $73.7 \%$ & \\
\hline \multirow{3}{*}{ First $_{T}$ Last $_{M}$} & 1 & 11 & 180 & $94.2 \%$ \\
\hline & 0 & 1,271 & 1,719 & $57.5 \%$ \\
\hline & Recall & $99.1 \%$ & $90.5 \%$ & \\
\hline \multirow{3}{*}{ First $_{M}$ Last $_{M}$} & 1 & 42 & 130 & $75.6 \%$ \\
\hline & 0 & 1,240 & 1,769 & $\mathbf{5 8 . 8 \%}$ \\
\hline & Recall & $96.7 \%$ & $93.2 \%$ & \\
\hline \multirow{3}{*}{ First $_{T}$ Last $_{T}$} & 1 & 76 & 1,090 & $93.5 \%$ \\
\hline & 0 & 1,206 & 809 & $\mathbf{5 9 . 9 \%}$ \\
\hline & Recall & $94.1 \%$ & $57.4 \%$ & \\
\hline \multirow{3}{*}{ Disjoint $_{M, T}$} & 1 & 246 & 256 & $51.0 \%$ \\
\hline & 0 & 1,036 & 1,643 & $61.3 \%$ \\
\hline & Recall & $80.8 \%$ & $86.5 \%$ & \\
\hline \multirow{3}{*}{ Disjoint $_{T, M}$} & 1 & 0 & 70 & $100.0 \%$ \\
\hline & 0 & 1,282 & 1,829 & $\mathbf{5 8 . 8 \%}$ \\
\hline & Recall & $100.0 \%$ & $96.3 \%$ & \\
\hline
\end{tabular}

\section{SUMMARY \& FUTURE WORK}

We have shown that it is possible to improve the detection of false-positive RFID tag reads in a distribution center process by alternating the existing and adding additional antennas to the portals. Two different setups called Satellite Portals and Transition Portals have been evaluated and compared to the usual Standard Portals. The read logic of the new portals has been mapped to a set of portal specific attributes which were used together with certain low-level reader data attributes proposed in [5] to generate three different classification algorithms. The Satellite Portal approach clearly outperformed the Standard Portal approach when it comes to the detection of moved pallets. The Transition Portal approach on the other was most suitable for the detection of static pallets, i.e., falsepositive RFID tag reads.

Although the results are very convincing it must be stated that the use of an additional RFID reader and 4 more antennas almost doubles the hardware costs. Therefore it must be evaluated on a project-basis if the additional costs justify a deployment or not.

Besides the generation of even more advanced algorithms it would be interesting to think of even better antenna setups maybe with the use of less antennas to decrease the price. The application of the proposed solution to different processes, i.e., incoming goods, electronic article surveillance and backroom shelf replenishment is imaginable and should be tested in the near future.
TABLE IX

Transition Portal Attribute Value Distribution

\begin{tabular}{|c|c|c|c|c|}
\hline Attribute & Value & Moved & Static & Precision \\
\hline \multirow{3}{*}{ Seen $_{\text {First }}$} & $\mathrm{D}$ & 1,191 & 1,022 & $53.8 \%$ \\
\hline & $\mathrm{T}$ & 108 & 1,370 & $92.7 \%$ \\
\hline & Recall & $91.7 \%$ & $57.3 \%$ & \\
\hline \multirow{3}{*}{ Seen $_{\text {Last }}$} & $\mathrm{D}$ & 127 & 732 & $85.2 \%$ \\
\hline & $\mathrm{T}$ & 1,172 & 1,660 & $58.6 \%$ \\
\hline & Recall & $90.2 \%$ & $69.4 \%$ & \\
\hline \multirow{3}{*}{ Seen $_{\text {Longer }}$} & $\mathrm{D}$ & 367 & 707 & $65.8 \%$ \\
\hline & $\mathrm{T}$ & 932 & 1,685 & $64.4 \%$ \\
\hline & Recall & $71.7 \%$ & $70.4 \%$ & \\
\hline \multirow{3}{*}{ First $_{D}$ Last $_{T}$} & 1 & 1,087 & 581 & $65.2 \%$ \\
\hline & No & 212 & 1,811 & $89.5 \%$ \\
\hline & Recall & $83.7 \%$ & $75.7 \%$ & \\
\hline \multirow{3}{*}{ First $_{T}$ Last $_{D}$} & 1 & 23 & 291 & $92.7 \%$ \\
\hline & 0 & 1,276 & 2,101 & $62.2 \%$ \\
\hline & Recall & $98.2 \%$ & $87.8 \%$ & \\
\hline \multirow{3}{*}{ First $_{D}$ Last $_{D}$} & 1 & 104 & 441 & $80.9 \%$ \\
\hline & 0 & 1,195 & 1,951 & $62.0 \%$ \\
\hline & Recall & $92.0 \%$ & $81.6 \%$ & \\
\hline \multirow{3}{*}{ First $_{T}$ Last $_{T}$} & 1 & 85 & 1079 & $92.7 \%$ \\
\hline & 0 & 1,214 & 1,313 & $52.0 \%$ \\
\hline & Recall & $93.5 \%$ & $54.9 \%$ & \\
\hline \multirow{3}{*}{ Disjoint $_{D, T}$} & 1 & 123 & 245 & $66.6 \%$ \\
\hline & 0 & 1,176 & 2,147 & $64.6 \%$ \\
\hline & Recall & $90.5 \%$ & $89.8 \%$ & \\
\hline \multirow{3}{*}{ Disjoint $_{T, D}$} & 1 & 7 & 96 & 93.2\% \\
\hline & 0 & 1,292 & 2,296 & $64.0 \%$ \\
\hline & Recall & $99.5 \%$ & $96.0 \%$ & \\
\hline
\end{tabular}

TABLE $X$

RESULTS OF THE CLASSIFICATION ALGORITHMS

\begin{tabular}{l|lrrr}
\hline Portal Type & Results & Moved & Static & Overall \\
\hline Standard Portals & Accuracy & $94.66 \%$ & $98.51 \%$ & $97.57 \%$ \\
& Error & $5.34 \%$ & $1.49 \%$ & $2.43 \%$ \\
\cline { 2 - 5 } Satellite Portals & Accuracy & $\mathbf{9 7 . 4 1 \%}$ & $99.29 \%$ & $\mathbf{9 9 . 0 4 \%}$ \\
& Error & $\mathbf{2 . 5 9 \%}$ & $0.71 \%$ & $\mathbf{0 . 9 6 \%}$ \\
\cline { 2 - 5 } Transition Portals & Accuracy & $89.62 \%$ & $\mathbf{9 9 . 5 2 \%}$ & $98.55 \%$ \\
& Error & $10.38 \%$ & $\mathbf{0 . 4 8 \%}$ & $1.45 \%$ \\
\hline
\end{tabular}

\section{ACKNOWLEDGMENT}

The authors would like to thank METRO Group and METRO Group Cash \& Carry for making their monitored low-level reader data available. Furthermore the authors would like to thank the team of IBM Global Business Services for valuable input and feedback.

\section{REFERENCES}

[1] D. C. Wyland, "RFID 101: The next big Thing for Management," Management Research News, vol. 29, no. 4, pp. 154-173, 2006.

[2] F. Thiesse, C. Floerkemeier, M. Harrison, F. Michahelles, and C. Roduner, "Technology, Standards, and Real-World Deployments of the EPC Network," IEEE Internet Computing, vol. 13, no. 2, pp. 36-43, 2009.

[3] R. Want, "The Magic of RFID," ACM Queue, vol. 2, no. 7, pp. 41-48, 2004.

[4] B. C. Hardgrave and R. Miller, RFID Technology and Applications. Cambridge, UK: Cambridge University Press, 2008, ch. RFID in the Retail Supply Chain: Issues and Opportunities.

[5] T. Keller, F. Thiesse, J. Kungl, and E. Fleisch, "Using low-level reader data to detect false-positive rfid tag reads," in Proceedings of the 2nd International Conference on the Internet of Things (IOT), Tokyo, Japan, November 2010.

[6] S. B. Miles, S. E. Sarma, and J. R. Williams, RFID Technology and Applications. Cambridge, UK: Cambridge University Press, 2008. 
[7] S. S. Chawathe, V. Krishnamurthy, S. Ramachandran, and S. Sarma, "Managing RFID Data," in Proceedings of the 30th International Conference on Very Large Data Bases (VLDB), Toronto, Canada, 2004, pp. 1189-1195.

[8] C. Floerkemeier and M. Lampe, "Issues with RFID Usage in Ubiquitous Computing Applications," in Proceedings of the 2nd International Conference on Pervasive Computing, Vienna, Austria, April 2004, pp. 188-193.

[9] Y. Bai, F. Wang, and P. Liu, "Efficiently filtering RFID Data Streams," in Proceedings of the 1st International Very Large Data Base Workshop on Clean Databases (CleanDB), Seoul, South Korea, September 2006, pp. $50-57$.

[10] B. Jiang, K. Fishkin, S. Roy, and M. Philipose, "I Sense a Disturbance in the Force: Unobtrusive long-range Detection of passive RFID Tag Motion," IEEE Transactions on Instrumentation and Measurement, vol. 55, no. 1, pp. 187-196, 2006.

[11] S. R. Jeffery, M. Garofalakis, and M. Franklin, "Adaptive Cleaning for RFID Data Streams," in Proceedings of the 32nd International Conference on Very Large Data Bases (VLDB), Seoul, South Korea, September 2006, pp. 163-174.

[12] Y.-J. Tu and S. Piramuthu, "Reducing False Reads in RFID-Embedded Supply Chains," Journal of Theoretical and Applied Electronic Commerce Research, vol. 3, no. 2, pp. 60-70, 2008.

[13] _ , "A Decision Support Model for Filtering RFID Read Data," in Proceedings of the 16th International Conference on Advanced Computing and Communications (ADCOM), Chennai, India, December 2008, pp. 221-224

[14] I. Mierswa, M. Wurst, R. Klinkenberg, M. Scholz, and T. Euler, "Yale: Rapid prototyping for complex data mining tasks," in $K D D$ '06: Proceedings of the 12th ACM SIGKDD international conference on Knowledge discovery and data mining, L. Ungar, M. Craven, D. Gunopulos, and T. Eliassi-Rad, Eds. New York, NY, USA: ACM, August 2006, pp. 935-940 